\title{
An Interpretation Of The Technical Efficiency As The "Best Possible Deviation" From The Conditions Defined By The Weak Axiom Of Profit Maximization
}

Said Gattoufi, (E-mail: gattoufi@squ.edu.om), Sultan Qaboos University, Oman Yuntong Wang, (E-mail: yuntong@uwindsor.ca), University of Windsor, Canada Arnold Reisman, (E-mail: arnoldreisman@sbcglobal.net), Reisman and Associates, Ohio Muhittin Oral, (E-mail: muhittin.oral@sabanciuniv.edu.tr), Sabanci University, Turkey

\begin{abstract}
This paper provides a characterization of the classical Charnes, Cooper and Rhodes' (CCR) model in Data Envelopment Analysis (DEA). The characterization is based on the Weak Axiom of Profit Maximization (WAPM) in Firm Theory. Efficiency measures for Decision Making Units (DMUs) provided by the classical CCR-DEA model are derived as measurements of deviations from the conditions prescribed by the Weak Axiom of Profit Maximization (WAPM).
\end{abstract}

\section{INTRODUCTION}

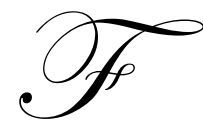

rom its very outset the Charnes, Cooper and Rhodes (1978) CCR-model in Data Envelopment Analysis (DEA) has enjoyed a high number and a high incidence of real-world applications. This can be observed via a casual perusal of the DEA based literature. However it happens to be a fact that is documented via content analysis of all DEA articles appearing between 1978 and 2001in Gattoufi et al. (2004). Moreover, the real world acceptance of DEA is in stark contrast to many other and older OR/MS sub-disciplines over their entire life-times, Reisman et. al. (1994, 1997a \& b), and (2001). Though the firmness of DEAs roots in the theory of mathematical programming is beyond question, this paper extends these roots into the fertile soil of economic theory. Efficiency measures for Decision Making Units (DMU's) provided by the original CCR-DEA model (1978), are herein derived as a natural extension of the Weak Axiom of Profit Maximization (WAPM) (Varian, 1992).

Economics and Operational Research have common interests as to several research fields, one of them being productivity analysis in general and Data Envelopment Analysis in particular. Forsund and Sarafoglou (2002) provides an excellent exposure about the origins of DEA. Although the origins of DEA are claimed to be in economics theory, it is a matter of fact that it is more popular in OR/MS community. Russell (1998) reports that:

Farrell (1957)'s contribution was itself ignored for more than two decades. It was rediscovered by Charnes, Cooper and Rhodes (1978), who referred to the mathematical programming method for determining the efficient frontier and measuring the technical efficiency as a deviation from that frontier as Data Envelopment Analysis (DEA), an appellation that seems to have stuck.

This paper provides a simple derivation of the original CCR model in DEA using the Weak Axiom of Profit Maximization in the theory of the firm suggested by Varian (1992). In another extension of Varian (1984)'s work, Banker and Maindiratta (1988) derive th DEA as corollary using a different approach by considering what production possibility set could rationalize the observed data, without specifically mention the CCR model. However, our approach is much simpler and straightforward as far as the DEA is concerned. 


\section{PARAMETRIC AND NON-PARAMETRIC APPROACHES TO EFFICIENCY ANALYSIS}

The concept of efficiency is a central concept in economic theory and full efficiency is defined as the attainment of Pareto optimality (Koopmans 1951). The efficiency reflects the degree of goodness with which economic units achieve their objectives. This raises issues of measuring efficiency and whether, indeed, an absolute measure of efficiency does exist.

There is an agreement in the existing literature, see for example Coelli et al. (2002), that the modern measurement of economic efficiency was introduced by Farrell (1957) who drew upon the work of Debreu (1951) and Koopmans (1951) to define a simple measure of a firm's efficiency. He proposed that the economic efficiency of a firm is a combination of its technical efficiency, which reflects its ability to obtain the maximal outputs from a given set of inputs, and its allocative efficiency, which reflects its ability to use inputs in optimal proportions given their respective prices.

In order to determine efficiency measures for firms, Farrell (1957) proposes to first identify an assumed existing efficient frontier using the production function. Then deviations from the efficient frontier have a natural interpretation as a measure of inefficiency with which economic units, or firms, pursue their technical or behavioral objectives.

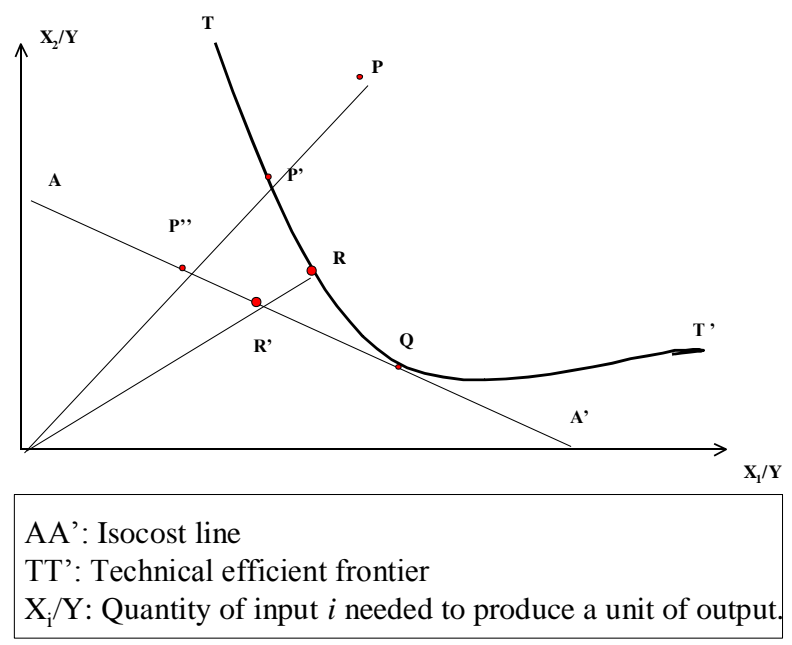

Figure 1: Technical and Allocative Efficient Frontiers

Figure 1 represents the efficient technical and allocative frontiers in the case of firms using two inputs to produce a single output. In this particular example, the economic units are competing in the same market using two inputs to produce a single output. The isoquant TT', defined by a specific function, determines all combinations of minimum inputs to produce a single unit of output. The curve represents the technically efficient frontier for the economic units considered. These are assumed to be homogeneous and to be competing under identical conditions. Furthermore they are presumed to have access to the same set of inputs with identical prices and to produce the same outputs. When the prices of inputs are considered, the line AA' represents the isocost line. Units P, Q and R are three different competing units. Projections on the axes of $\mathrm{P}$ and $\mathrm{Q}$ determine the level of inputs used to produce a single unit of output.

The unit $\mathrm{P}$ is neither technically nor allocatively efficient. The virtual units $\mathrm{P}$ ' represent the technically efficient and the allocatively efficient targets for unit P. Unit R is technically but not allocatively efficient. Units P', and R' are not feasible points, they represent the cost penalty associated with the allocative mistake made at points P' and $\mathrm{R}$ respectively. Unit $\mathrm{Q}$ is economically efficient since no allocative penalty is associated and is on the technically 
efficient frontier.

In real life, the function defining the efficient frontier is often not known explicitly. It is typically approximated in order to define the efficient frontier. Farrell (1957) suggested the use of either (i) a non-parametric piecewise linear convex form or (ii) a parametric function. DEA belongs to the first class of above methods. Altered forms of the Cobb-Douglas function predominantly constitute the second class.

The parametric approaches specify an explicit functional form for the efficient frontier. This function is assumed to be reflecting the production process, usually takes an altered form of Cobb-Douglas function, and reflecting the relationships between inputs and outputs within the production process. An econometric model is used to estimate the parameters of the underlying function. This model, unlike classical ones, has the particularity of having an error term with two components. The first component of the error term, a white noise, reflects the classical randomness of econometric models. The second component reflects the deviation from the efficient frontier. Different functional forms of the latter component of the error term are proposed in the literature, see for example Battese and Coelli (1992, 1995), Resti (1998) and Coelli (1996) for a specific software.

The non-parametric approach, mainly DEA with its derivations and extensions, is a mathematical programming based methodology for efficiency analysis. The formulation leads to a linear program with an objective function reflecting the best efficiency level that the economic unit being evaluated can reach. The constraints of the linear program define a piecewise-linear frontier of a convex simplex that forms the efficient frontier. Charnes and Cooper (1985), one of the most cited references in DEA literature, provides an excellent presentation of DEA.

\section{DATA ENVELOPMENT ANALYSIS: THE CHARNES-COOPER-RHODES (CCR) MODEL}

Productivity measurement and efficiency evaluation methods in economics, business and engineering are ratio-based approaches to assess the performance of economic units, e.g., firms, products, production systems or, in the parlance of DEA, DMUs. Output-to-input ratio measures are commonly used in these fields to evaluate performance of such units. However, most of these approaches are used to provide absolute measures of performance. DEA, although it is also a ratio-based approach, has the distinguished characteristic of always providing relative measures of performance for each DMU in a set of such DMUs. The DMUs involved in the analysis are assumed to be homogeneous and competing in the same market while utilizing the same set of inputs to produce the same set of outputs.

The best performers among the DMUs considered are used to define what is called the efficient frontier. Specifically this frontier is defined as a convex combination of the best performers, considered to be fully efficient. Deviations from the efficient frontier are interpreted as measures of inefficiency for the remaining DMUs. A virtually efficient target, belonging to the efficient frontier, is identified for each inefficient DMU. The radial deviation from the efficient virtual target is interpreted as a measure of inefficiency. Thus the ratio of the radial distance of the virtual efficient target to the radial distance of the corresponding DMU defines the efficiency measure. Its complement is the unit measure of its inefficiency. In less technical and more concrete terms, the efficiency of a given DMU is measured, (in an input oriented DEA), by comparing the inputs it needs to those needed by the most efficient virtual DMU in order to produce an equivalent amount of output. Conventionally, a fully efficient DMU is given $\boldsymbol{l}$ (unity) as a measure of efficiency and all efficiency coefficients have non-zero values. The mathematical form of the original DEA model, the ratio form known as the CCR model, is as follows: 


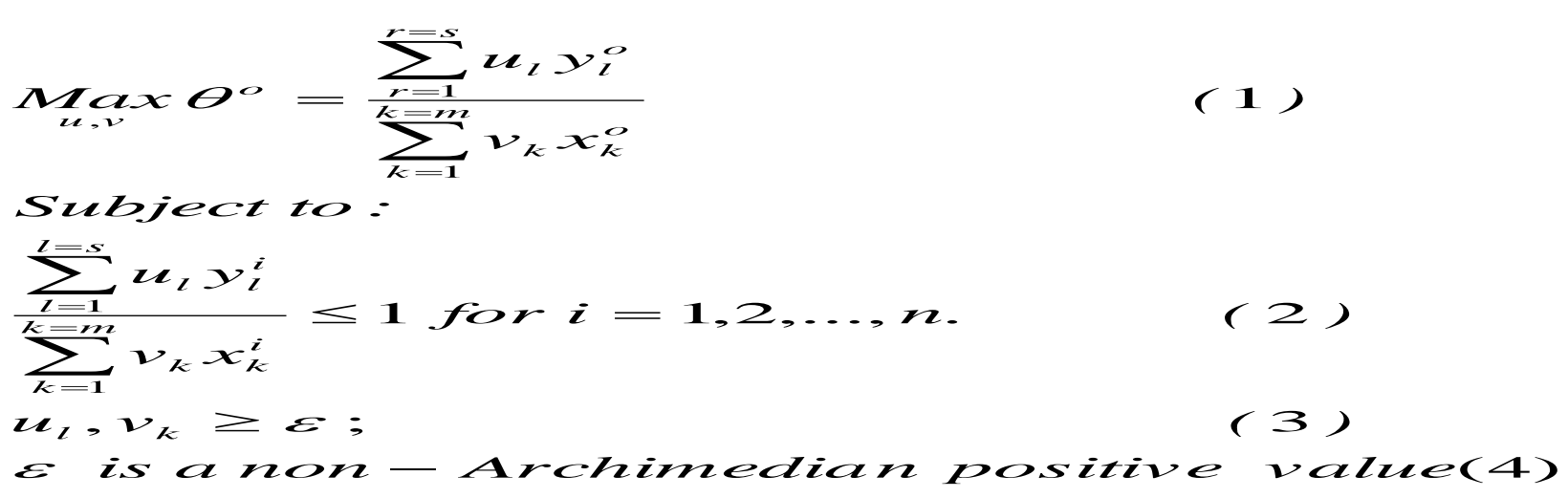

This model considers a set of $\boldsymbol{n}$ DMUs competing in the same market. The production process requires a set of $\boldsymbol{m}$ inputs to produce $\boldsymbol{s}$ outputs. The subscript ' $o$ ' is generic taking any value ' $\mathrm{o}$ ' $=1,2, \ldots, \mathrm{n}$. It refers to the DMU being evaluated, called the base-DMU.

The objective function described by Equation (1) measures the efficiency of the base-DMU via the maximum value of its ratio. The optimal value of the objective function is obtained through the generation of a virtual input, (a combination of all real inputs), and a virtual output, (a combination of all real outputs). Moreover, the relative weights given to the real factors (inputs and outputs) in the definition of the optimal virtual input and output are represented by the coefficients obtained at the optimum. The decision variables are in fact the coefficients $(u, v)$ for the virtual factors.

The $\boldsymbol{m} * \boldsymbol{n}$ matrix $\boldsymbol{x}$ specifies the data regarding the inputs: $\boldsymbol{x}_{i j}$ is the quantity of input (i) used by DMU $(j)$ in the production process. Correspondingly, the $\boldsymbol{s} * \boldsymbol{n}$ matrix $\boldsymbol{y}$ specifies the data regarding the outputs: $\boldsymbol{y}_{\boldsymbol{r} j}$ is the quantity of output $(r)$ produced by DMU $(j)$ in the production process. The set of constraints in (2) states that all efficiency coefficients are constrained to be unity (1) or less, the value normalized for full efficiency. The classical non-negativity condition is replaced by condition (3) involving a non-Archimedian value. To ensure that the variables of the models, the weights for inputs and outputs, are accorded some worth, they are constrained to be not only positive but also greater than any positive real number. This is obtained by considering as lower bound for these variables a nonArchimedian infinitesimal smaller than any positive real number. For further discussion regarding the above see Cooper et al. (2000).

The main drawback with this form is its unboundedness of optimal solutions since if $(u, v)$ represents a solution then for any positive real value $\square \square(\square u \square \square v)$ as well is a solution. Fractional linear programming suggests considering a representative from each class of solutions. This can be obtained by normalizing and transforming the original model into the following linear program, called the multiplier form:

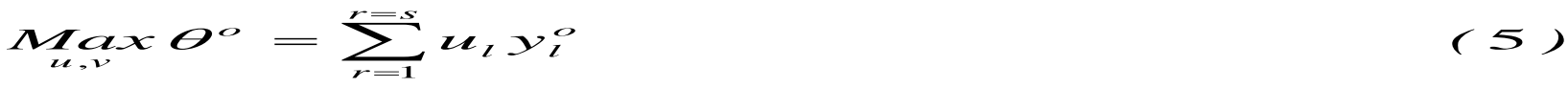

$$
\begin{aligned}
& \text { subject to:- } \\
& \left.\sum_{i=1}^{l=s} \mu_{i} y_{i}^{i}-\sum_{k=1}^{k=m} \nu_{k} x_{k}^{i} \leq 1 \text { for } i=1,2, \ldots, n \text { ( } 6\right) \\
& \sum_{k=1}^{k=m} v_{k} x_{k}^{0}=1 \quad \text { (7) } \\
& \mu_{1}, V_{k} \geq \text {; ( } 8 \text { ) } \\
& \text { E is a non - Archinedian positive value(9) }
\end{aligned}
$$


For further details about non-Archimedian parameter and fractional linear programming see Charnes and Cooper (1962), Cooper et al. (2000) and Coelli et al (2000).

\section{CHARACTERIZING THE CCR DEA MODEL BY THE WEAK AXIOM OF PROFIT MAXIMIZATION}

\section{The Classical Theory Of A Firm}

Classical theory (Mas-Colell et al., 1995; Varian, 1992) characterizes a firm by the set of all possible production plans the firm can execute, namely the Possible Production Set (PPS). A firm's performance, under given economic conditions, is evaluated solely on the choices it makes from its PPS. On the other hand, DEA focuses on measures of performance relative to the firm's peers. These two perspectives reflect the differences between economic theory and management science. In economic theory, firms are treated similar to consumers. Any firm-specific internal details are ignored in the competitive market. In management science, on the other hand, firms are treated as live organizations, within which management makes ongoing decisions at various levels.

\section{The Weak Axiom Of Profit Maximization (WAPM)}

A basic assumption underlying economic theory of firm behavior is that a firm acts so as to maximize its profit. Accepting this assumption leads to accepting all its implications. The Weak Axiom of Profit Maximization (WAPM) happens to be a well established (see Varian, 1992) restriction that is imposed by the Profit Maximization assumption.

To describe WAPM, consider a given DMU (or firm) from a finite set of DMUs and a list of observed price vectors $p^{t}=\left(p_{x}^{t}, p_{y}^{t}\right) ; t=1,2, \ldots, n$ and its respective production plans $\left(-x^{t}, y^{t}\right) ; t=1,2, \ldots, n$ chosen by the firm under the above price vectors. The collection of $\left\{p^{t},\left(-x^{t}, y^{t}\right) ; t=1,2, \ldots, n \cdot\right\}$ is called data.

If the firm is maximizing its profit, the observed chosen production vector $\left(-x^{t}, y^{t}\right)$ for a given price vector $p^{t}$ must generate a profit at least as much as, or greater than, the profit generated by any alternative production plan $\left(-x^{s}, y^{s}\right) ; s \neq t$ available to the firm using the given price vector. Although the alternative production plans are not all identified, some of them are described by the set of vectors: $\left(-x^{s}, y^{s}\right) ; s=1,2, \ldots, n$. Hence, a necessary condition for profit maximization is:

$$
p^{t}\left(-x^{t}, y^{t}\right) \geq p^{t}\left(-x^{s}, y^{s}\right) ; \text { for any } s=1,2, \ldots, n
$$

The condition in (10) is called the Weak Axiom of Profit Maximization (WAPM). In fact, it can be shown that if a set of data satisfies WAPM it is always possible to find a technology (PPS) for which the observed data are profit-maximizing choices (see Varian, 1992). Thus, WAPM exhausts all the implications of profit-maximization assumption.

\section{Relating The CCR-DEA Model To The Classical Theory Of A Firm}

Consider a finite set of $\boldsymbol{n}$ homogeneous DMUs, regarded as a set of $\boldsymbol{n}$ firms competing in the same environment. Assume that all firms have the same PPS, using a common set of $\boldsymbol{m}$ inputs to produce a common set of $\boldsymbol{s}$ outputs. This means that any DMU is able to attain any production plan belonging to the common PPS. However, every DMU is characterized by a single (chosen and attained) production plan. 
To be specific, each DMU (i), for $\mathrm{i}=1,2, \ldots, \mathrm{n}$, is characterized by a production plan $y^{i}=\left(-x_{1}^{i},-x_{2}^{i}, \ldots,-x_{m}^{i}, y_{1}^{i}, y_{2}^{i}, \ldots, y_{s}^{i}\right)$ which belongs to the common set of all realizable production plans, namely, the PPS::

$$
Y=\left\{\left(-x_{1},-x_{2}, \ldots,-x_{m}, y_{1}, y_{2}, \ldots, y_{s}\right)\right\}
$$

The negative sign is used to distinguish inputs from outputs. Given the required data for all DMUs, the objective next is to measure their respective relative efficiencies.

We maintain the traditional assumptions on the technology. We assume that $\mathrm{Y}$ exhibits a constant returns to scale, i.e., $y \in Y$ implies $\alpha y \in Y$ for any scalar $\alpha \geq 0$. Assume also that $\mathrm{Y}$ is convex. That is if $y, y^{\prime} \in Y$ and $\alpha \in[0,1]$, then $\alpha y+(1-\alpha) y^{\prime} \in Y$.

\section{Derivation Of The CCR Model From WAPM}

Consider first a specific DMU, which is to be comparatively evaluated against the remaining DMUs in a given set. Assume that DMU(o) chooses the production plan described by the vector:

$$
\left(-x_{1}^{o},-x_{2}^{o}, \ldots,-x_{m}^{o}, y_{1}^{o}, y_{2}^{o}, \ldots, y_{s}^{o}\right) \in Y
$$

Let $p^{o}=\left(p_{x}^{o}, p_{y}^{o}\right)$ be the shadow price vector (endogenously determined) for DMU(o) with $p_{x}^{o}=\left(p_{x_{1}}^{o}, p_{x_{2}}^{o}, \ldots, p_{x_{m}}^{o}\right) \geq 0$ and $p_{y}^{o}=\left(p_{y_{1}}^{o}, p_{y_{2}}^{o}, \ldots, p_{y_{s}}^{o}\right) \geq 0$, where $p_{x_{k}}^{o}$ is the unit price for input $x_{k} ; k=1,2, \ldots, m$ and $p_{y_{l}}^{o}$ is the unit price for output $y_{l} ; l=1,2, \ldots, s$. Assuming that DMU(o) satisfies the WAPM leads to:

$$
-\sum_{k=1}^{k=m} p_{x_{k}}^{o} x_{k}^{o}+\sum_{l=1}^{l=s} p_{y_{l}}^{o} y_{l}^{o} \geq-\sum_{k=1}^{k=m} p_{x_{k}}^{o} x_{k}^{i}+\sum_{l=1}^{l=s} p_{y_{l}}^{o} y_{l}^{i} \text { for } i=1,2, \ldots, n
$$

where $\left(-x_{1}^{i},-x_{2}^{i}, \ldots,-x_{m}^{i}, y_{1}^{i}, y_{2}^{i}, \ldots, y_{s}^{i}\right), i=1,2, \ldots, n$ are DMU(i)'s production plans.

This means that the base DMU(o) chooses the production plan defined by (12) because it generates, for the given price vector, the highest profit when compared with the profit generated by any of the remaining production plans belonging to the production set, including production plans chosen by other DMUs.

Clearly, DMU(o) could have chosen any one of the remaining production plans. Yet it chose not to, thereby leaving them for the other DMUs.

Without loss of generality, we normalize the profit of $\mathrm{DMU}(\mathrm{o})$ generated from the chosen production plan $\left(-x_{1}^{o},-x_{2}^{o}, \ldots,-x_{m}^{o}, y_{1}^{o}, y_{2}^{o}, \ldots, y_{s}^{o}\right)$ to be zero (the long-run economic profit).

That is, we assume:

$-\sum_{k=1}^{k=m} p_{x_{k}}^{o} x_{k}^{o}+\sum_{l=1}^{l=s} p_{y_{l}}^{o} y_{l}^{o}=0$ 
This implies, from WAPM, that the profits generated by other realizable production plans $\left(-x_{1}^{i},-x_{2}^{i}, \ldots,-x_{m}^{i}, y_{1}^{i}, y_{2}^{i}, \ldots, y_{s}^{i}\right) ; i=1,2, \ldots, n ;$ are less than or equal to zero. Therefore

$-\sum_{k=1}^{k=m} p_{x_{k}}^{o} x_{k}^{i}+\sum_{l=1}^{l=s} p_{y_{l}}^{o} y_{l}^{i} \leq \mathrm{O}$

or

$$
\frac{\sum_{l=1}^{l=s} p_{y_{l}}^{o} y_{l}^{i}}{\sum_{k=1}^{k=m} p_{x_{k}}^{o} x_{k}^{i}} \leq 1 \text { for } i=1,2, \ldots, n
$$

and particularly

$$
\frac{\sum_{l=1}^{l=s} p_{y_{l}}^{o} y_{l}^{o}}{\sum_{k=1}^{k=m} p_{x_{k}}^{o} x_{k}^{o}}=1
$$

However, for the given price vector $p^{o}=\left(p_{x}^{o}, p_{y}^{o}\right)$ condition (13) specified by WAPM may not hold or such price vector $p^{o}=\left(p_{x}^{o}, p_{y}^{o}\right)$ may not even exist. In the first case, it might be because the real production plan $\left(-x_{1}^{o},-x_{2}^{o}, \ldots,-x_{m}^{o}, y_{1}^{o}, y_{2}^{o}, \ldots, y_{s}^{o}\right)$ adopted by the base DMU(o) was not based solely on profit maximization (yet the production plan could be technically efficient). In the second case, the firm simply (mistakenly) chose an inefficient plan. Additionally, in this case, the real data represented by the current production plan may not be optimal in any economic environment (characterized by price vectors).

Since economic theory of the firm presumes firms (DMUs) to be profit-maximizers, the WAPM must be satisfied by the firms' production choices. As we mentioned before, the converse holds as well, namely, the firm must be a profit-maximizer if it always satisfies the WAPM. What can be said about a firm's behavior if the WAPM is violated and/or where the observed data do not satisfy the WAPM? Certainly, the profit-maximization hypothesis or assumption is not going to be rejected. The profit maximization hypothesis has been very successful as a theoretical model for approximating or describing the firm's behavior. The deviation or violation of the WAPM can be interpreted in many ways. A firm that violates the WAPM may have other objectives in addition to profit maximization (indeed this is often the case). The violation of the WAPM can also be interpreted as a consequence of the bounded rationality characterized by incomplete information about technical and/or economic environments the firm is dealing with and more.

Whatever are the reasons, we propose that for a given set of firms, a firm's relative efficiency should be measured in terms of how best it minimizes its deviation from the conditions prescribed by WAPM. This leads us to establish the following main result of this paper.

Theorem 1: Under the profit maximization postulate of the firm, the CCR-DEA model is characterized by the best possible deviation from WAPM.

Proof: If firms violate the WAPM, the assumption that firms reduce as much as possible their deviation from the conditions defined by the WAPM is equivalent to the assumption that, given all the technical or economic information 
in hand, they are maximizing their profits.

The reduction, as much as possible, of the deviation from the optimal conditions defined by the WAPM gives rise to the profit maximization or efficient production plans. This is then equivalent to saying that the present choice of the production plan generates the highest realizable profit level under the constraints that all other production plans by the the other DMUs are at most at their optimal levels (efficient production plans).

To properly formulate and apply this idea of optimal deviation in the context of the CCR-DEA model, let the maximum realizable profit be normalized to zero. More specifically, the maximum profit that any DMU can achieve for any given price vector is normalized to be equal to zero. It is clear and with no loss of generality assuming that all DMUs have at most zero profits as far as WAPM is concerned. For the base DMU(o), if its profit is zero at that given price vector it is classified as a fully efficient DMU. Obviously in this case the WAPM is satisfied. Alternatively, if DMU(o)'s profit is strictly less than zero (it can not be positive by the above assumption), we will try to find a best price vector $p^{*}$ (economic environment) such that $\mathrm{DMU}(\mathrm{o})$ maximizes its profit from the chosen production plan $\left(-x_{1}^{o},-x_{2}^{o}, \ldots,-x_{m}^{o}, y_{1}^{o}, y_{2}^{o}, \ldots, y_{s}^{o}\right)$ under the condition that the profit from any other production plan by other DMUs is at most zero. This captures the assumption that firms or DMUs minimize their deviation from from the conditions imposed by WAPM. Formally, we have:

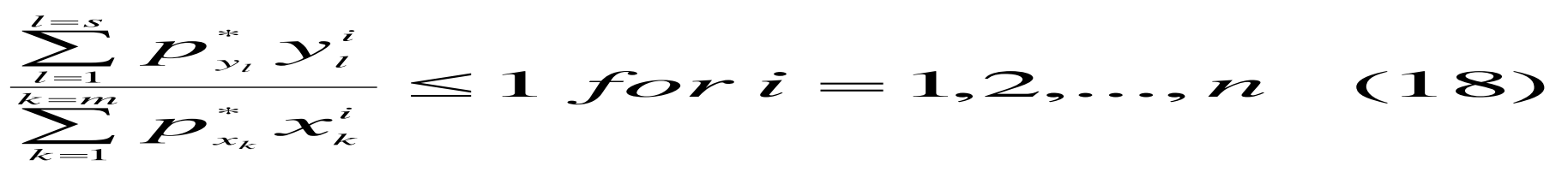

and
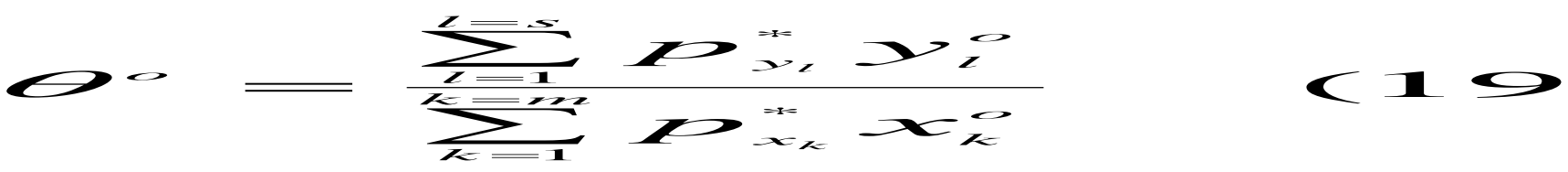

is maximized.

However, this is exactly what CCR states. That is:

$$
\operatorname{Max}_{p \rightarrow 0}=\frac{\sum_{i=1}^{l=s} P_{y_{i}}^{*} y_{i}^{0}}{\sum_{k=1}^{k=m} P_{x_{k}}^{*} x_{k}^{0}} \quad \text { (20) }
$$

Subject to:
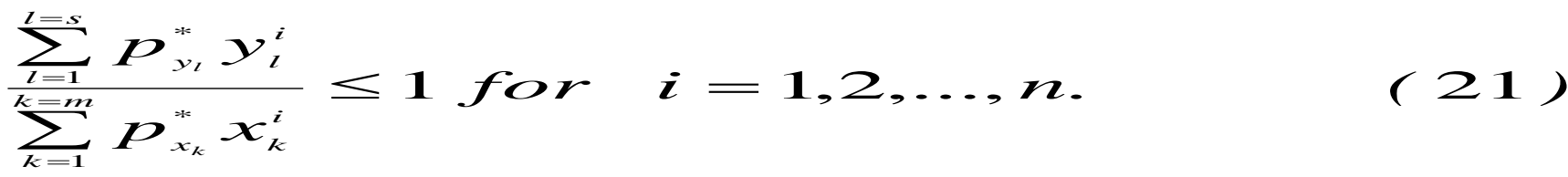

\section{CONCLUSIONS}

In this paper, the $C C R-D E A$ model was derived from the classical economic theory of the firm. Specifically, since the WAPM characterizes the firm's profit maximization behavior assumption, any departure from the WAPM indicates the firm's departure from the profit optimization assumption (not necessarily being the firm's intention), 
which further indicates that the firm is operating inefficiently. The relative departure from the WAPM therefore can be used to measure the relative inefficiency of the firm, based on the firm's own production plan as well as other counterparts or competing firms' plans. This naturally induces the CCR-DEA model to evaluate the relative efficiency between firms under the Profit Maximization postulate.

In fact, Varian $(1984,1990)$ proposed goodness-of-fit measures for the violations of relevant conditions defined by the optimization models on firms, consumers, and so on. However, in testing the optimization model of a firm, he focuses on a single firm. He suggests to first test, based on the observed data, if the firm violates the WAPM, and if it does, define a measure to evaluate the significance of the deviation from conditions defined by the WAPM. However, the purpose of this work is to measure performance relative efficiency of a number of firms given that each firm has a single set of data. For a given firm, the departure (if there is any) from the WAPM measures its relative efficiency with regard to other firms (or their production plans since here a firm is uniquely characterized by a production plan). Therefore, this paper extends Varian (1984, 1990).

Traditionally, the applications of WAPM involve, as stated by Varian $(1984,1990)$, "the activity analysis of a single firm". This work represents an extension to multi-firm analysis. In particular, it is interesting to see that the original DEA model, the CCR model, can be simply derived from WAPM.

\section{REFERENCES}

1. Banker, R. D. and Maindiratta, A. (1988). Nonparametric Analysis of Technical Efficiencies in Production, Econometrica 56, 1315-1332.

2. Battese, G. E. and Coelli, T. J. (1992). Frontier production functions, technical efficiency and panel data: with application to paddy farmers in India, Journal of Productivity Analysis 3, 153-169.

3. Battese, G. E. and Coelli, T. J. (1995). A model for technical inefficiency effects in a stochastic frontier production function for panel data, Empirical Economics 20, 325-332.

4. Charnes, A. and Cooper, W.W. (1962). Programming with linear fractional Functionals, Naval Research Logistics Quarterly 9, 181-186.

5. Charnes, A. and Cooper, W. W. (1985). Preface to topics in Data Envelopment Analysis, Annals of Operations Research 2(1) (1985) 59-94.

6. Charnes, A., Cooper, W. W., and Rhodes, E. (1978). Measuring the efficiency of decision making units, European Journal of Operational Research 2, 429-444.

7. Coelli, T. (1996), A Guide to DEAP2.1: A Data Envelopment Analysis (Computer) program, Working Paper, Centre for Efficiency and Productivity Analysis, University of New England, Australia.

8. Cooper, W. W., Seiford, M. L., and Kaoru, T. (2000), Data Envelopment Analysis: A comprehensive text with models, applications, references and DEA-solver software, Kluwer Academic Publishers, Dordrecht, The Netherlands.

9. Debreu, G. (1951). The coefficient of resource utilisation, Econometrica 19, 273-292.

10. Farrell, M.J. (1957). The measurement of productive efficiency, Journal of Royal Statistical Society-Series A CXX (1957) 253-281.

11. Forsund, F. R. and N. Sarafoglou. (2002). On the origins of Data Envelopment Analysis. Journal of Productivity Analysis 17, 23-40.

12. Gattoufi, S., Oral, M., and Reisman, A. (2001). Content Analysis of Data Envelopment Analysis Literature, Sabanci University Working Paper.

13. Koopmans, T.C. (1951). An analysis of production as an efficient combination of activities in T. C. Koopmans, Ed., Activity Analysis of Production and Allocation, Cowles Commission for Research in Economics, Monograph13, Wiley, New York, 1951.

14. Mas-Colell, A., Whinston, M. D., and Green, J. R. (1995). Microeconomic Theory, Oxford University Press, New York.

15. Reisman, A. and Kirschnick, F. (1995). Research strategies used by OR/MS workers as shown by an analysis of papers in flagship journals, Operations Research 43/5, 731-740.

16. Reisman, A., Kumar, A., Motwani, J., and Cheng, C. H. (1997a). Cellular Manufacturing: A Statistical 
Review of The Literature (1965-1995), Operations Research 45/4, 508-520.

17. Reisman, A., Kumar, A., and Motwani, J. (1997b). Flowshop Scheduling/Sequencing Research: A Statistical Review of the Literature 1952-1994, IEEE Transactions on Engineering Management 44/3, 316-329.

18. Russel, R. R. (1998). Distance Functions in Consumer and Producer Theory in R. Fare, S. Grosskopf, and R. R. Russel (eds), Index Numbers: Essays in Honour of Sten Malmquist. Boston/London/Dordrecht, Kluwer Academic publishers, 7-90.

19. Varian, H. R. (1984). The Nonparametric Approach to Production Analysis, Econometrica 54, 579-597.

20. Varian, H. R. (1990). Goodness-of-fit in optimizing models, Journal of Econometrics 46 125-140.

21. Varian, H. R. (1992). Microeconomic Analysis, Third Edition, W. W. Norton \& Company, New York, 1992.

\section{NOTES}

